\title{
A QCD Debye mass in a broad temperature range
}

\author{
M. Laine, ${ }^{1}$ P. Schicho $\odot,{ }^{1}$ and Y. Schröder $\circledast^{2}$ \\ ${ }^{1}$ AEC, Institute for Theoretical Physics, University of Bern, Sidlerstrasse 5, 3012 Bern, Switzerland \\ ${ }^{2}$ Grupo de Cosmología y Partículas Elementales, Universidad del Bío-Bío, Casilla 447, Chillán, Chile
}

(Received 26 November 2019; published 30 January 2020)

\begin{abstract}
The Debye mass sets a scale for the screening of static charges and the scattering of fast charges within a gauge plasma. Inspired by its potential cosmological applications, we determine a QCD Debye mass at two-loop order in a broad temperature range ( $1 \mathrm{GeV}-10 \mathrm{TeV})$, demonstrating how quark mass thresholds get smoothly crossed. Along the way, integration-by-parts identities pertinent to massive loops at finite temperature are illuminated.
\end{abstract}

DOI: 10.1103/PhysRevD.101.023532

\section{INTRODUCTION}

If two test charges are put a distance $r$ apart within a plasma, they influence each other with a force which is weaker than the Coulomb force in vacuum, as a result of the screening caused by the light plasma particles. The potential then takes a Yukawa form, $-\alpha e^{-m_{\mathrm{E}} r} / r$, where the parameter $m_{\mathrm{E}}$ may be called an electric or a Debye mass. In a relativistic plasma, it is of order $m_{\mathrm{E}} \sim g T$, where $T$ is the temperature and $g$ is a gauge coupling.

In the present paper, we focus on strong interactions, such that $g$ is the coupling of the SU(3) gauge force. Standard applications of the QCD Debye mass can be found in the physics of heavy ion collision experiments. However, the temperatures reached there $(T \ll 1 \mathrm{GeV})$ are so low that it is questionable whether perturbative tools are viable. Here, we rather take $T \gtrsim 1 \mathrm{GeV}$ and consider the possible role of the QCD Debye mass in cosmology.

Given that strong interactions are in thermal equilibrium in a broad temperature range, QCD does not normally play a prominent role in cosmology. However, exceptions can be envisaged. ${ }^{1}$ For instance, it has become popular to consider scenarios in which dark matter is but the lightest among the particles of a larger dark sector. Then, it is conceivable that the dark sector may also contain particles charged under QCD (cf. e.g,. Ref. [1] for a review of one such framework). At high temperatures, the pair annihilation of the QCD-charged particles would be modified by

\footnotetext{
${ }^{1}$ None of the contexts listed here is "urgent," as they are related to yet-to-be-discovered beyond the Standard Model physics; nevertheless, we hope that, put together, they can motivate a well-defined QCD computation.

Published by the American Physical Society under the terms of the Creative Commons Attribution 4.0 International license. Further distribution of this work must maintain attribution to the author(s) and the published article's title, journal citation, and DOI. Funded by SCOAP ${ }^{3}$.
}

Debye screening [2]. Charged particles also experience a thermal mass shift, known as the Salpeter correction in plasma physics, $\Delta M_{\mathrm{T}} \sim-\alpha m_{\mathrm{E}} / 2$, which can have a, relatively speaking, large effect if narrow degeneracies are present in the dark sector.

Another possible application concerns the decay of heavy particles, for instance, right-handed neutrinos in leptogenesis. In this case, it is important to know how fast the decay products (some of which could be hadronic, produced through the Higgs channel) equilibrate kinetically [3]. This requires large-angle scattering, again sensitive to Debye screening [4]. Another relevant rate, namely, that of decoherence of the decay products, originates from a difference of small-angle scatterings mediated by colormagnetic and color-electric fields, whereby it is nonvanishing at $\mathcal{O}(\alpha T)$ thanks to $m_{\mathrm{E}} \neq 0$ (cf. e.g., Ref. [5]).

A third application of Debye masses is that they play a role in dimensionally reduced descriptions of the electroweak phase transition [6]. In particular, the QCD Debye mass could make a noticeable appearance if some colored scalar field is light enough to participate in the transition dynamics (cf. e.g., Refs. [7,8]).

This paper is organized as follows. The definition of a Debye mass is subtle beyond leading order, so we start by specifying the concept adopted in Sec. II. The main steps and methods of the computation are described in Sec. III, and results are presented in Sec. IV. We conclude in Sec. V, relegating the evaluation of massive one-loop and two-loop sum integrals to the Appendix.

\section{FORMULATION OF THE PROBLEM}

As mentioned at the beginning of the Introduction, the leading-order definition of a Debye mass can be related to the Yukawa screening of a static potential or, equivalently, to the thermal mass that color-electric fields obtain. In $\mathrm{SU}\left(N_{\mathrm{c}}\right)$ gauge theory with $N_{\mathrm{f}}$ massless fermions, the classic result reads [9] 


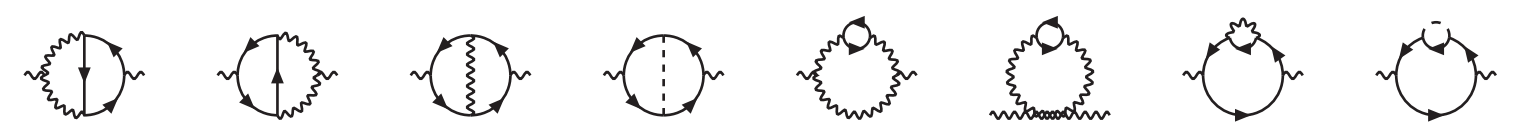

FIG. 1. Fermionic two-loop contributions to the gluon two-point function. Wiggly lines denote gluons, solid lines denote quarks, and dashed lines denote Higgs bosons.

$$
m_{\mathrm{E}}^{2}=g^{2} T^{2}\left(\frac{N_{\mathrm{c}}}{3}+\frac{N_{\mathrm{f}}}{6}\right)+\mathcal{O}\left(g^{3} T^{2}\right)
$$

When we go beyond leading order, the definition of a Debye mass is no longer unique. One possibility is to define it as the inverse of a spatial correlation length related to some gauge-invariant operator [10]. This way, the Debye mass becomes nonperturbative at next-to-leading order [11]. However, correlation lengths depend strongly on the quantum numbers of the operator chosen. There are also other nonperturbative possibilities, related, e.g., to modeling the behavior of the static potential at intermediate distances [12].

A different strategy is to define the Debye mass as a "matching coefficient" of a low-energy description, specifically of a dimensionally reduced effective theory $[13,14]$. There are a number of advantages with this strategy. One is that the definition is then "universal," with the same value appearing as an ingredient in the computation of many different correlation lengths [15], or even of dynamical rates [5]. Another is that as a matching coefficient $m_{\mathrm{E}}^{2}$ is only sensitive to the hard scales that have been integrated out, and therefore perturbative by construction. In fact, the result is known analytically up to three-loop order in pure Yang-Mills theory $[16,17]$ and shows remarkable convergence down to low temperatures. Fermionic effects are for this definition currently known up to two-loop order in the massless limit [18] and up to one-loop level in the massive case [9]. The purpose of the current study is to extend the two-loop result for $m_{\mathrm{E}}^{2}$ to include massive fermions. ${ }^{2}$

To be explicit, the action of the dimensionally reduced effective theory, often called "electrostatic QCD” (EQCD), reads

$S_{\mathrm{EQCD}} \equiv \int_{X}\left\{\frac{1}{4} F_{i j}^{a} F_{i j}^{a}+\frac{1}{2} \mathcal{D}_{i}^{a b} A_{0}^{b} \mathcal{D}_{i}^{a c} A_{0}^{c}+\frac{m_{\mathrm{E}}^{2}}{2} A_{0}^{a} A_{0}^{a}+\ldots\right\}$,

where we are employing Euclidean conventions, $\int_{X} \equiv$ $\frac{1}{T} \int \mathrm{d}^{d} \mathbf{x}, \quad d=3-2 \epsilon, \quad F_{i j}^{a} \equiv \partial_{i} A_{j}^{a}-\partial_{j} A_{i}^{a}+g_{\mathrm{E}} f^{a b c} A_{i}^{b} A_{j}^{c}$, $\mathcal{D}_{i}^{a b} \equiv \delta^{a b} \partial_{i}-g_{\mathrm{E}} f^{a b c} A_{i}^{c}$, and $A_{0}^{a}$ is an adjoint scalar field.

\footnotetext{
${ }^{2}$ We note in passing that another analogous matching coefficient is the gauge coupling of the dimensionally reduced theory, denoted by $g_{\mathrm{E}}^{2}$, but its determination is technically more challenging. For pure Yang-Mills theory, results are available up to three-loop level, but only in somewhat incomplete numerical form [19-21]. Massless fermions have been included up to twoloop level [22], and mass effects have been included up to oneloop level [23].
}

To determine $m_{\mathrm{E}}^{2}$, it is convenient to use the background field gauge [24] as a probe. We compute the temporal two-point function with a purely spatial external momentum,

$\Pi_{00}(\mathbf{p}) \equiv \Pi_{\mathrm{E}}\left(p^{2}\right)=\sum_{n=1}^{\infty} g_{\mathrm{B}}^{2 n} \Pi_{\mathrm{E} n}\left(p^{2}\right), \quad p \equiv|\mathbf{p}|$.

Here, $g_{\mathrm{B}}^{2}$ is the bare coupling, which is subsequently expressed in terms of the renormalized coupling $g^{2}$. The computation within full QCD (or the Standard Model) is matched onto a computation within the effective theory, the latter also reexpanded as a perturbative series in $g^{2}$. However, employing dimensional regularization and Taylor expanding in external momentum, the latter computation gives a vanishing result, given that no scales appear in the propagators. Therefore, the matching coefficient is directly given by a Taylor-expanded full theory computation, after accounting for different field normalizations (or wave function corrections) within the full and effective theories,

$m_{\mathrm{E}}^{2}=g_{\mathrm{B}}^{2} \Pi_{\mathrm{E} 1}(0)+g_{\mathrm{B}}^{4}\left[\Pi_{\mathrm{E} 2}(0)-\Pi_{\mathrm{E} 1}(0) \Pi_{\mathrm{E} 1}^{\prime}(0)\right]+\mathcal{O}\left(g_{\mathrm{B}}^{6}\right)$

\section{MAIN STEPS OF THE COMPUTATION}

The Feynman diagrams required for determining the two-loop fermionic contributions to $m_{\mathrm{E}}^{2}$ are shown in Fig. 1 (we do not show gluonic diagrams, as our results for them agree with Ref. [18]). Apart from vertices involving the strong gauge coupling $g^{2}$, we have for illustration also included effects from the top Yukawa coupling $h_{t}^{2}$, even if in practice these are small. ${ }^{3}$

The computation is carried out by employing the gauge propagator

$$
\left\langle A_{\mu}^{a}(P) A_{\nu}^{b}(Q)\right\rangle \equiv \delta(P+Q) \delta^{a b}\left[\frac{\delta_{\mu \nu}}{P^{2}}+\frac{\xi P_{\mu} P_{\nu}}{P^{4}}\right],
$$

\footnotetext{
${ }^{3}$ For the latter set, only the scalar coupling to the physical Higgs mode has been kept. The couplings to the Goldstone modes lead to gauge-dependent contributions which can only be included if the $\mathrm{SU}_{\mathrm{L}}(2) \times \mathrm{U}_{\mathrm{Y}}(1)$ gauge bosons are incorporated as well; however, those effects are numerically very small.
} 
where $\mathscr{E}_{P} \delta(P)=1$ and $\mathscr{E}_{P} \equiv T \sum_{p_{n}} \mu^{3-d} \int \frac{\mathrm{d}^{d} \mathbf{p}}{(2 \pi)^{d}}$ is a bosonic Matsubara sum integral. We keep $\xi$ as a general gauge parameter (in the background field gauge, it also appears in the cubic and quartic gauge vertices [24]), verifying that it cancels exactly at the end.

After carrying out the Wick contractions, the result can be expressed in terms of the "master" sum integrals

$$
\begin{aligned}
& Z_{j ; i}^{r} \equiv \bigcup_{\{P\}} \frac{p_{n}^{r}}{\left(P^{2}+m_{i}^{2}\right)^{j}}, \quad \hat{Z}_{j ; 0}^{r} \equiv \bigvee_{P} \frac{p_{n}^{r}}{\left(P^{2}\right)^{j}}, \\
& Z_{j k l ; i}^{r s} \equiv \bigvee_{\{P, Q\}} \frac{p_{n}^{r} q_{n}^{s}}{\left(P^{2}+m_{i}^{2}\right)^{j}\left(Q^{2}+m_{i}^{2}\right)^{k}\left[(P-Q)^{2}\right]^{l}},
\end{aligned}
$$

where $P \equiv\left(p_{n}, \mathbf{p}\right)$ and $p_{n}$ is a Matsubara frequency. The sum integral $\mathfrak{W}_{\{P\}}$ signifies that $p_{n}$ is fermionic, i.e., $p_{n} \equiv$ $\pi T(2 n+1)$ with $n \in \mathbb{Z}$. After renaming variables, the set of two-loop masters can then be chosen to consist of $Z_{j k l ; i}^{20}$, $Z_{j k l ; i}^{11}$, and $Z_{j k l ; i} \equiv Z_{j k l ; i}^{00}$. In rare cases, we add a mass $m_{h}$ on the bosonic line and indicate this with the index $h$. The mass index $i$ is omitted in intermediate results if this can be done without the danger of confusion.

As far as one-loop results are concerned, we need [cf. Eq. (2.4)]

$$
\begin{aligned}
\Pi_{\mathrm{E} 1}(0)= & (d-1)^{2} N_{\mathrm{c}} \hat{Z}_{1 ; 0}-2 \sum_{i=1}^{N_{\mathrm{f}}}\left[(d-1) Z_{1 ; i}+2 m_{i}^{2} Z_{2 ; i}\right], \\
\partial_{m_{i}^{2}} \Pi_{\mathrm{E} 1}(0)= & 2 \sum_{i=1}^{N_{\mathrm{f}}}\left[(d-3) Z_{2 ; i}+4 m_{i}^{2} Z_{3 ; i}\right], \\
\Pi_{\mathrm{E} 1}^{\prime}(0)= & -\left[\frac{d^{2}-5 d+28}{6}-\xi(d-3)\right] N_{\mathrm{c}} \hat{Z}_{2 ; 0} \\
& +\frac{1}{3} \sum_{i=1}^{N_{\mathrm{f}}}\left[(d-1) Z_{2 ; i}+4 m_{i}^{2} Z_{3 ; i}\right] .
\end{aligned}
$$

Equation (3.5) is relevant because $\Pi_{\mathrm{E} 1}$ is originally expressed as a function of bare quark masses, which are expanded as $m_{\mathrm{B} i}^{2}=m_{i}^{2}\left[1+\frac{3 h_{i}^{2}-12 g^{2} C_{\mathrm{F}}}{2(4 \pi)^{2} \epsilon}+\mathcal{O}\left(g^{4}\right)\right]$, where $C_{\mathrm{F}} \equiv\left(N_{\mathrm{c}}^{2}-1\right) /$ $\left(2 N_{\mathrm{c}}\right)$. In practice, Yukawa couplings $h_{i}$ other than $h_{t}$ are omitted. Similarly, the bare gauge coupling is renormalized as $g_{\mathrm{B}}^{2}=g^{2}\left[1+\frac{g^{2}}{(4 \pi)^{2} \epsilon}\left(-\frac{11 N_{\mathrm{c}}}{3}+\frac{2}{3} \sum_{i=1}^{N_{\mathrm{f}}}\right)+\mathcal{O}\left(g^{4}\right)\right]$.

The two-loop diagrams yield products of the one-loop masters of Eq. (3.2) as well as genuine two-loop masters defined according to Eq. (3.3). All numerators can be eliminated from one-loop masters by making use of $Z_{j+1 ; i}^{r+2}=-m_{i}^{2} Z_{j+1 ; i}^{r}+\left(1-\frac{d}{2 j}\right) Z_{j ; i}^{r}$. This produces

$$
\begin{aligned}
\Pi_{\mathrm{E} 2}(0)= & -N_{\mathrm{c}}^{2}(d-1)^{2}(d-3)(1-\xi) \hat{Z}_{1 ; 0} \hat{Z}_{2 ; 0} \\
& +N_{\mathrm{c}} \sum_{i=1}^{N_{\mathrm{f}}}\left\{2(d-1)(d-3)(1-\xi) Z_{1 ; i} \hat{Z}_{2 ; 0}+4 m_{i}^{2}\left[Z_{112}-\xi(d-3) Z_{2 ; i} \hat{Z}_{2 ; 0}\right]\right. \\
& \left.+8 m_{i}^{2}\left[Z_{221}^{11}+2\left(Z_{212}^{11}-Z_{212}^{20}\right)+4\left(Z_{113}^{11}-Z_{113}^{20}\right)\right]\right\} \\
& +C_{\mathrm{F}} \sum_{i=1}^{N_{\mathrm{f}}}\left\{2(d-1)\left[\hat{Z}_{1 ; 0}-Z_{1 ; i}\right]\left[(d-3) Z_{2 ; i}+4 m_{i}^{2} Z_{3 ; i}\right]+8 m_{i}^{2}\left[Z_{211}-2 Z_{221}^{11}-4 Z_{311}^{20}\right]\right\} \\
& -\frac{h_{t \mathrm{~B}}^{2}}{g_{\mathrm{B}}^{2}}\left\{\left[Z_{1 ; t}-\hat{Z}_{1 ; h}\right]\left[(d-3) Z_{2 ; t}+4 m_{t}^{2} Z_{3 ; t}\right]+\left(4 m_{t}^{2}-m_{h}^{2}\right)\left[Z_{211 ; t h}-2 Z_{221 ; t h}^{11}-4 Z_{311 ; t h}^{20}\right]\right\} .
\end{aligned}
$$

The set of masters can now be reduced by making use of integration-by-parts (IBP) identities [25], generalized to finite temperature [26]. First, inspecting

$$
0=\bigcup_{\{P, Q\}} \sum_{i=1}^{d} \frac{\partial}{\partial p_{i}} \frac{p_{i} \pm q_{i}}{\left(P^{2}+m^{2}\right)^{j}\left(Q^{2}+m^{2}\right)^{k}\left[(P-Q)^{2}\right]^{l}}
$$

and taking linear combinations leads to relations which permit us to eliminate all quadratic powers of $p_{n}$,

$$
\begin{aligned}
Z_{(j+1) k l}^{20}= & \frac{1}{2 j}\left\{(2 j+k-d) Z_{j k l}-2 m^{2}\left[j Z_{(j+1) k l}+k Z_{j(k+1) l}\right]+k\left[Z_{(j-1)(k+1) l}-Z_{j(k+1)(l-1)}-2 Z_{j(k+1) l}^{11}\right]\right\}, \\
Z_{j k(l+1)}^{20}= & \frac{1}{2 l}\left\{(l-k) Z_{j k l}+2 k m^{2} Z_{j(k+1) l}\right. \\
& \left.+k\left[Z_{j(k+1)(l-1)}-Z_{(j-1)(k+1) l}+2 Z_{j(k+1) l}^{11}\right]+l\left[Z_{(j-1) k(l+1)}-Z_{j(k-1)(l+1)}+2 Z_{j k(l+1)}^{11}\right]\right\} .
\end{aligned}
$$


Second, if we choose indices leading to two independent representations of some $Z_{j k l}^{20}$, we can establish relations between $Z_{j k l}^{11}$. Considering $Z_{212}^{20}$ this way, we obtain from Eqs. (3.9) and (3.10) the identity

$$
2\left(Z_{221}^{11}+2 Z_{212}^{11}\right)=Z_{2 ; i}\left(2 \hat{Z}_{2 ; 0}-Z_{2 ; i}\right)-2 m^{2}\left(Z_{221}+2 Z_{212}\right)-(d-2) Z_{112}
$$

By using Eq. (3.9) in order to eliminate $Z_{311}^{20}$ and Eq. (3.10) to eliminate $Z_{113}^{20}$ and $Z_{212}^{20}$ and inserting subsequently Eq. (3.11), we can remove all numerators from the sum integrals of Eq. (3.7), leading to

$$
\begin{aligned}
\Pi_{\mathrm{E} 2}(0) \supset & N_{\mathrm{c}} \sum_{i=1}^{N_{\mathrm{f}}}\left\{2(d-1)(d-3)(1-\xi) Z_{1 ; i} \hat{Z}_{2 ; 0}+4 m_{i}^{2}[2-\xi(d-3)] Z_{2 ; i} \hat{Z}_{2 ; 0}\right. \\
& \left.+4 m_{i}^{2}\left[(d-5) Z_{112}-2 m_{i}^{2} Z_{221}-\left(Z_{2 ; i}\right)^{2}\right]\right\} \\
& +C_{\mathrm{F}} \sum_{i=1}^{N_{\mathrm{f}}}\left\{2(d-1)\left[\hat{Z}_{1 ; 0}-Z_{1 ; i}\right]\left[(d-3) Z_{2 ; i}+4 m_{i}^{2} Z_{3 ; i}\right]\right. \\
& \left.+8 m_{i}^{2}\left[(d-5) Z_{211}+2 m_{i}^{2}\left(Z_{221}+2 Z_{311}\right)+\left(Z_{2 ; 1}\right)^{2}\right]\right\} .
\end{aligned}
$$

Remarkably, IBP relations also exist between masters without any numerators. In this way, we can eliminate $Z_{221}, Z_{211}$, and $Z_{311}$ in favor of $Z_{111}, Z_{112}$, and $Z_{212}$. The latter set is convenient, as it turns out that $Z_{111}$ appears with zero coefficient in $d$ dimensions, and $Z_{212}$ can be obtained from $Z_{112}$ through a mass derivative. Thereby, only one irreducible master, $Z_{112}$, remains to be determined in detail (cf. Appendix A 2). ${ }^{4}$

The relations needed, originally found via our FORM [27] implementation of Laporta-type reduction [28], read

$$
\begin{gathered}
Z_{211}=-\frac{(d-3) Z_{111}}{4 m^{2}}+\frac{\left(Z_{2 ; i}\right)^{2}}{2(d-2)}, \\
Z_{221}+2 Z_{311}=\frac{(d-3)(d-5) Z_{111}}{8 m^{4}}+\frac{Z_{2 ; i}\left[8 m^{2} Z_{3 ; i}-(d-3) Z_{2 ; i}\right]}{4(d-2) m^{2}}, \\
Z_{221}=\frac{(d-2)(d-5) Z_{112}}{4 m^{2}}+(d-4) Z_{212}+\frac{Z_{2 ; i}\left(2 \hat{Z}_{2 ; 0}-Z_{2 ; i}\right)}{4 m^{2}} .
\end{gathered}
$$

Defining $\Delta_{\mathrm{P}} \equiv P^{2}+m^{2}$ and $\delta_{\mathrm{P}} \equiv P^{2}$, Eqs. (3.13) and (3.15) can be verified by setting $s=1$ and $s=2$, respectively, in the relation

$$
\begin{aligned}
0= & \mathcal{Y}_{\{P, Q\}} \sum_{i=1}^{d} \frac{\partial}{\partial p_{i}}\left\{\frac{(d-2 s) p_{i}}{\Delta_{P} \Delta_{Q} \delta_{P-Q}^{s}}+\frac{2 p_{n}\left(p_{n} q_{i}-q_{n} p_{i}\right)}{\Delta_{P} \Delta_{Q}^{2} \delta_{P-Q}^{s}}-\frac{p_{i}}{\Delta_{P} \Delta_{Q}^{2} \delta_{P-Q}^{s-1}}+\frac{p_{i}-q_{i}}{\Delta_{Q}^{2} \delta_{P-Q}^{s}}\right\} \\
& +\mathcal{Y}_{\{P, Q\}} \sum_{i=1}^{d} \frac{\partial}{\partial q_{i}}\left\{\frac{(d-2 s) p_{i}}{\Delta_{P} \Delta_{Q} \delta_{P-Q}^{s}}-\frac{2 p_{n}\left(p_{n} q_{i}-q_{n} p_{i}\right)}{\Delta_{P}^{2} \Delta_{Q} \delta_{P-Q}^{s}}-\frac{(s-1) p_{i}}{\Delta_{P}^{2} \Delta_{Q} \delta_{P-Q}^{s-1}}\right\},
\end{aligned}
$$

whereas Eq. (3.14) can be established by taking a mass derivative of Eq. (3.13).

\footnotetext{
${ }^{4}$ Taken on its own, the master $Z_{112}$ is IR divergent. However, the matching coefficient $m_{\mathrm{E}}^{2}$ as a whole is IR safe by construction. For a proper cancellation of IR divergences, all masters need to be consistently evaluated with dimensional regularization, which regularizes both their IR and UV divergences.
} 
Inserting Eqs. (3.13)-(3.15), the $C_{\mathrm{F}}$ part gets factorized, and Eq. (3.12) reduces to

$$
\begin{aligned}
\Pi_{\mathrm{E} 2}(0) \supset & N_{\mathrm{c}} \sum_{i=1}^{N_{\mathrm{f}}}\left\{2(d-1)(d-3) Z_{1 ; i} \hat{Z}_{2 ; 0}+2 m_{i}^{2} Z_{2 ; i}\left(2 \hat{Z}_{2 ; 0}-Z_{2 ; i}\right)\right. \\
& \left.-2 \xi(d-3) \hat{Z}_{2 ; 0}\left[(d-1) Z_{1 ; i}+2 m_{i}^{2} Z_{2 ; i}\right]-2(d-4) m_{i}^{2}\left[(d-5) Z_{112}+4 m_{i}^{2} Z_{212}\right]\right\} \\
& +C_{\mathrm{F}} \sum_{i=1}^{N_{\mathrm{f}}}\left[(d-3) Z_{2 ; i}+4 m_{i}^{2} Z_{3 ; i}\right]\left\{2(d-1)\left[\hat{Z}_{1 ; 0}-Z_{1 ; i}\right]+\frac{8 m_{i}^{2} Z_{2 ; i}}{d-2}\right\} .
\end{aligned}
$$

Adding to Eq. (3.17) the contributions from Eqs. (3.4) and (3.6) according to Eq. (2.4) and reinstalling the $N_{\mathrm{c}}^{2}$ and $h_{t}^{2}$ parts of Eq. (3.7), we write the result in terms of $\overline{\mathrm{MS}}$-renormalized couplings as

$$
\begin{aligned}
m_{\mathrm{E}}^{2}= & g^{2}\left[N_{\mathrm{c}} \Phi^{(1)}+\sum_{i=1}^{N_{\mathrm{f}}} \Phi_{i}^{(2)}\right] \\
& +g^{4}\left[N_{\mathrm{c}}^{2} \Phi^{(3)}+\sum_{i=1}^{N_{\mathrm{f}}}\left(N_{\mathrm{c}} \Phi_{i}^{(4)}+C_{\mathrm{F}} \Phi_{i}^{(5)}\right)+\sum_{i, j=1}^{N_{\mathrm{f}}} \Phi_{i}^{(2)} \Phi_{j}^{(6)}\right]+g^{2} h_{t}^{2} \Phi^{(7)}+\mathcal{O}\left(g^{6}\right) .
\end{aligned}
$$

The various functions employed in Eq. (3.18) read

$$
\begin{aligned}
& \Phi^{(1)}=(d-1)^{2} N_{\mathrm{c}} \hat{Z}_{1 ; 0}, \\
& \Phi_{i}^{(2)}=-2\left[(d-1) Z_{1 ; i}+2 m_{i}^{2} Z_{2 ; i}\right], \\
& \Phi^{(3)}=(d-1)^{2} \hat{Z}_{1 ; 0}\left[\frac{\left(d^{2}-11 d+46\right) \hat{Z}_{2 ; 0}}{6}-\frac{11}{(4 \pi)^{2} 3 \epsilon}\right],
\end{aligned}
$$
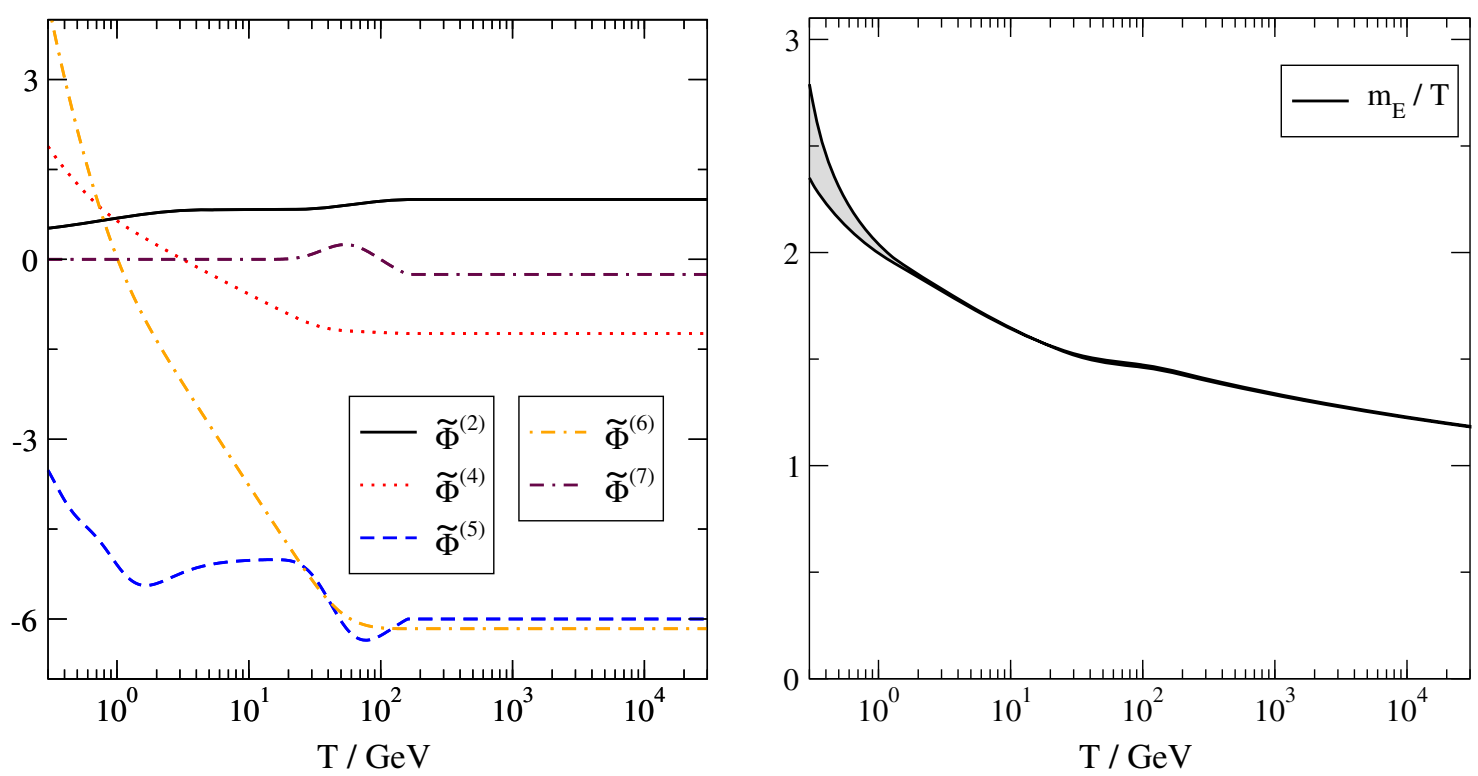

FIG. 2. Left: the coefficients $\tilde{\Phi}^{(2)} \equiv \sum_{i=1}^{N_{\mathrm{f}}} \Phi_{i}^{(2)} / T^{2}, \tilde{\Phi}^{(4,5)} \equiv \sum_{i=1}^{N_{\mathrm{f}}}(4 \pi)^{2} \Phi_{i}^{(4,5)} / T^{2}, \tilde{\Phi}^{(6)} \equiv \sum_{j=1}^{N_{\mathrm{f}}}(4 \pi)^{2} \Phi_{j}^{(6)}$, and $\tilde{\Phi}^{(7)} \equiv(4 \pi)^{2} \Phi^{(7)} / T^{2}$ that parametrize Eq. (3.18), evaluated with $\bar{\mu}=2 \pi T$. Right: the QCD Debye mass as a function of the temperature. The gray band originates from varying the renormalization scale in the range $\bar{\mu}=(0.5 \ldots 2.0) \times 2 \pi T$ and gives an indication of the magnitude of higherorder corrections. The "hard scale" with which $m_{\mathrm{E}}$ can be compared is approximately $2 \pi T$. The plateaulike feature centered around $T \sim 70 \mathrm{GeV}$ originates from crossing the top mass threshold. 


$$
\begin{aligned}
& \Phi_{i}^{(4)}= {\left[(d-1) Z_{1 ; i}+2 m_{i}^{2} Z_{2 ; i}\right]\left[\frac{22}{(4 \pi)^{2} 3 \epsilon}-\frac{\left(d^{2}-5 d+28\right) \hat{Z}_{2 ; 0}}{3}\right] } \\
&+(d-1)^{2} \hat{Z}_{1 ; 0}\left[\frac{2}{(4 \pi)^{2} 3 \epsilon}-\frac{\left.(d-1) Z_{2 ; i}+4 m_{i}^{2} Z_{3 ; i}\right]}{3}\right] \\
&+2(d-1)(d-3) Z_{1 ; i} \hat{Z}_{2 ; 0}+2 m_{i}^{2} Z_{2 ; i}\left(2 \hat{Z}_{2 ; 0}-Z_{2 ; i}\right)-2 m_{i}^{2}(d-4)\left[(d-5) Z_{112}+4 m_{i}^{2} Z_{212}\right], \\
& \Phi_{i}^{(5)}=-\left[(d-3) Z_{2 ; i}+4 m_{i}^{2} Z_{3 ; i}\right]\left[\left(\frac{12}{(4 \pi)^{2} \epsilon}-\frac{8 Z_{2 ; i}}{d-2}\right) m_{i}^{2}+2(d-1)\left(Z_{1 ; i}-\hat{Z}_{1 ; 0}\right)\right], \\
& \Phi_{j}^{(6)}=-\frac{1}{3}\left[(d-1) Z_{2 ; j}+4 m_{j}^{2} Z_{3 ; j}-\frac{2}{(4 \pi)^{2} \epsilon}\right], \\
& \Phi^{(7)^{m}} \stackrel{m_{h} \ll m_{t}}{\approx}-\left[(d-3) Z_{2 ; t}+4 m_{t}^{2} Z_{3 ; t}\right]\left[\left(\frac{4 Z_{2 ; t}}{d-2}-\frac{3}{(4 \pi)^{2} \epsilon}\right) m_{t}^{2}+Z_{1 ; t}-\hat{Z}_{1 ; 0}\right] .
\end{aligned}
$$

These are gauge independent, i.e., no $\xi$ appears, and also finite after the insertion of the masters from the Appendix; i.e., no $1 / \epsilon^{2}$ or $1 / \epsilon$ appears. For $\Phi^{(7)}$, we have approximated the result by considering the limit $m_{h} \ll m_{t}$, as this leads to the same basis as for the pure QCD contributions. This overestimates the magnitude of $\Phi^{(7)}$, but given that its effect is small even then (cf. Fig. 2), the approximation can be considered conservative.

\section{RESULTS}

Our final results for the coefficients in Eq. (3.18) are obtained by inserting the master sum-integrals from Appendix A into Eqs. (3.19)-(3.25) and by then expanding the expressions up to $\mathcal{O}\left(\epsilon^{0}\right)$. Denoting $\int_{p} \equiv \int \frac{\mathrm{d}^{3} \mathbf{p}}{(2 \pi)^{3}}, \omega_{p_{i}} \equiv$ $\sqrt{p^{2}+m_{i}^{2}}$ and $n_{\mathrm{F}}(\omega) \equiv 1 /[\exp (\omega / T)+1]$, we find

$$
\begin{aligned}
& \Phi^{(1)} \stackrel{d=3-2 \epsilon}{=} \frac{T^{2}}{3}, \\
& \Phi_{i}^{(2)} \stackrel{d=3-2 \epsilon}{=} 2 \int_{p} \frac{n_{\mathrm{F}}\left(\omega_{p_{i}}\right)}{\omega_{p_{i}}}\left(2+\frac{m_{i}^{2}}{p^{2}}\right), \\
& \Phi^{(3)} \stackrel{d=3-2 \epsilon}{=} \frac{22 T^{2}}{9(4 \pi)^{2}}\left[\ln \left(\frac{\bar{\mu} e^{\gamma_{\mathrm{E}}}}{4 \pi T}\right)+\frac{5}{22}\right] \text {, } \\
& \Phi_{i}^{(4)} \stackrel{d=3-2 \epsilon}{=} \frac{T^{2}}{9}\left[\int_{p} \frac{n_{\mathrm{F}}\left(\omega_{p_{i}}\right)}{p^{2} \omega_{p_{i}}}-\frac{2}{(4 \pi)^{2}} \ln \left(\frac{\bar{\mu}^{2}}{m_{i}^{2}}\right)\right] \\
& +\frac{44}{3(4 \pi)^{2}}\left[\ln \left(\frac{\bar{\mu} e^{\gamma_{\mathrm{E}}}}{4 \pi T}\right)+\frac{1}{2}\right] \int_{p} \frac{n_{\mathrm{F}}\left(\omega_{p_{i}}\right)}{\omega_{p_{i}}}\left(2+\frac{m_{i}^{2}}{p^{2}}\right)-\frac{8}{(4 \pi)^{2}} \int_{p} \frac{n_{\mathrm{F}}\left(\omega_{p_{i}}\right)}{\omega_{p_{i}}} \\
& +\frac{m_{i}^{2} T^{2}}{18}\left\{\int_{p} \frac{n_{\mathrm{F}}\left(\omega_{p_{i}}\right)}{p^{2} \omega_{p_{i}}^{3}}+\frac{1}{T} \int_{p} \frac{n_{\mathrm{F}}\left(\omega_{p_{i}}\right)\left[1-n_{\mathrm{F}}\left(\omega_{p_{i}}\right)\right]}{p^{2} \omega_{p_{i}}^{2}}\right\}-\frac{m_{i}^{2}}{2}\left\{\int_{p} \frac{n_{\mathrm{F}}\left(\omega_{p_{i}}\right)}{p^{2} \omega_{p_{i}}}\right\}^{2} \\
& +\int_{p, q} \frac{m_{i}^{2}}{8 \omega_{p_{i}} \omega_{q_{i}}}\left(\frac{1}{p^{2}}+\frac{1}{q^{2}}\right)\left\{\frac{\left[n_{\mathrm{F}}\left(\omega_{p_{i}}\right)+n_{\mathrm{F}}\left(\omega_{q_{i}}\right)\right]^{2}}{\left(\omega_{p_{i}}+\omega_{q_{i}}\right)^{2}}-\frac{\left[n_{\mathrm{F}}\left(\omega_{p_{i}}\right)-n_{\mathrm{F}}\left(\omega_{q_{i}}\right)\right]^{2}}{\left(\omega_{p_{i}}-\omega_{q_{i}}\right)^{2}}\right\}, \\
& \Phi_{i}^{(5)} \stackrel{d=3-2 \epsilon}{=}-\frac{m_{i}^{2}}{2}\left\{\int_{p} \frac{n_{\mathrm{F}}\left(\omega_{p_{i}}\right)}{p^{2} \omega_{p_{i}}^{3}}+\frac{1}{T} \int_{p} \frac{n_{\mathrm{F}}\left(\omega_{p_{i}}\right)\left[1-n_{\mathrm{F}}\left(\omega_{p_{i}}\right)\right]}{p^{2} \omega_{p_{i}}^{2}}\right\} \\
& \times\left\{\frac{T^{2}}{3}+\frac{12 m_{i}^{2}}{(4 \pi)^{2}}\left[\ln \left(\frac{\bar{\mu}^{2}}{m_{i}^{2}}\right)+\frac{4}{3}\right]+4 \int_{q} \frac{n_{\mathrm{F}}\left(\omega_{q_{i}}\right)}{\omega_{q_{i}}}\left(1-\frac{m_{i}^{2}}{q^{2}}\right)\right\},
\end{aligned}
$$




$$
\begin{aligned}
& \Phi_{j}^{(6)} \stackrel{d=3-2 \epsilon}{=} \frac{1}{3}\left\{\int_{q} \frac{n_{\mathrm{F}}\left(\omega_{q_{j}}\right)}{q^{2} \omega_{q_{j}}}-\frac{2}{(4 \pi)^{2}} \ln \left(\frac{\bar{\mu}^{2}}{m_{j}^{2}}\right)+\frac{m_{j}^{2}}{2}\left[\int_{q} \frac{n_{\mathrm{F}}\left(\omega_{q_{j}}\right)}{q^{2} \omega_{q_{j}}^{3}}+\frac{1}{T} \int_{q} \frac{n_{\mathrm{F}}\left(\omega_{q_{j}}\right)\left[1-n_{\mathrm{F}}\left(\omega_{q_{j}}\right)\right]}{q^{2} \omega_{q_{j}}^{2}}\right]\right\}, \\
& \Phi^{(7)} \stackrel{m_{h} \ll m_{t}}{\approx}-\frac{m_{t}^{2}}{2}\left\{\int_{p} \frac{n_{\mathrm{F}}\left(\omega_{p_{t}}\right)}{p^{2} \omega_{p_{t}}^{3}}+\frac{1}{T} \int_{p} \frac{n_{\mathrm{F}}\left(\omega_{p_{t}}\right)\left[1-n_{\mathrm{F}}\left(\omega_{p_{t}}\right)\right]}{p^{2} \omega_{p_{t}}^{2}}\right\} \\
& \times\left\{\frac{T^{2}}{12}-\frac{3 m_{t}^{2}}{(4 \pi)^{2}}\left[\ln \left(\frac{\bar{\mu}^{2}}{m_{t}^{2}}\right)+\frac{7}{3}\right]+\int_{q} \frac{n_{\mathrm{F}}\left(\omega_{q_{t}}\right)}{\omega_{q_{t}}}\left(1+\frac{2 m_{t}^{2}}{q^{2}}\right)\right\},
\end{aligned}
$$

where $m_{i}$ refer to $\overline{\mathrm{MS}}$ masses evaluated at the renormalization scale $\bar{\mu}$. In the limit $m_{i} \ll T$, the coefficients go over into

$$
\begin{aligned}
& \Phi_{i}^{(2)} \stackrel{m_{i} \ll T}{\approx} \frac{T^{2}}{6}, \\
& \Phi_{i}^{(4)} \stackrel{m_{i} \ll T}{\approx} \frac{7 T^{2}}{9(4 \pi)^{2}}\left[\ln \left(\frac{\bar{\mu} e^{\gamma_{\mathrm{E}}}}{4 \pi T}\right)-\frac{8 \ln 2}{7}+\frac{9}{14}\right], \\
& \Phi_{i}^{(5)} \stackrel{m_{i} \ll T}{\approx}-\frac{T^{2}}{(4 \pi)^{2}}, \\
& \Phi_{j}^{(6)} \stackrel{m_{j} \ll T}{\approx}-\frac{4}{3(4 \pi)^{2}}\left[\ln \left(\frac{\bar{\mu} e^{\gamma_{\mathrm{E}}}}{\pi T}\right)-\frac{1}{2}\right], \\
& \Phi^{(7)} \stackrel{m_{t, h} \ll T}{\approx}-\frac{T^{2}}{4(4 \pi)^{2}},
\end{aligned}
$$

reproducing in the first four cases the expressions obtained in Ref. [18]. For $m_{i} \gg \pi T$, all terms containing $n_{\mathrm{F}}$ are exponentially suppressed.

For a numerical evaluation, we set $\alpha_{\mathrm{s}}\left(m_{\mathrm{Z}}\right) \simeq 0.118$ [29] and evolve $g^{2}(\bar{\mu})$ in both directions with five-loop running [30-33], changing $N_{\mathrm{f}}$ when a threshold is crossed at $\bar{\mu} \simeq m_{i}$ and including effects from the top Yukawa up to three-loop order [34,35]. Quark masses are likewise evolved at five-loop level [36,37], including effects from the top Yukawa as indicated below Eq. (3.6). ${ }^{5}$ To account for temperature-dependent tadpole corrections proportional to $T^{2}$, the Higgs expectation value and $m_{i}$ are further scaled as $v_{\mathrm{T}} \simeq v_{0} \operatorname{Re} \sqrt{1-(T / 160 \mathrm{GeV})^{2}}$, where $v_{0} \simeq 246 \mathrm{GeV}$ and the crossover temperature has been adopted from

\footnotetext{
${ }^{5}$ Running quark masses become ambiguous at $\bar{\mu} \gtrsim m_{\mathrm{Z}}$, given that corrections to the Higgs vacuum expectation value from weak interactions are partly gauge dependent. That said, the $\mathrm{SU}_{\mathrm{L}}(2) \times \mathrm{U}_{\mathrm{Y}}(1)$ gauge effects are numerically very small compared with QCD corrections, as already alluded to in footnote 3, so we do not enter into a more detailed discussion of this topic here.
}

Ref. [38] rather than from a perturbative computation. As the top quark Yukawa coupling plays a minor role, we have resorted to two-loop running for $h_{t}^{2}$, with the initial condition $h_{t}^{2}\left(m_{\mathrm{Z}}\right) \simeq 0.95$ and running taking place only for $\bar{\mu}>m_{\mathrm{Z}}$. The initial value of the running top mass (before applying thermal rescaling) is estimated as $m_{t}\left(m_{\mathrm{Z}}\right) \simeq$ $h_{t}\left(m_{\mathrm{Z}}\right) v_{0} / \sqrt{2} \simeq 169.5 \mathrm{GeV}$, whereas those of the quartic Higgs and electroweak couplings, which affect the running of $h_{t}^{2}$, are $\lambda\left(m_{\mathrm{Z}}\right) \simeq 0.145$ and $g_{1}^{2}\left(m_{\mathrm{Z}}\right) \simeq 0.128$, $g_{2}^{2}\left(m_{\mathrm{Z}}\right) \simeq 0.425$, respectively. The renormalization scale is set to $\bar{\mu}=(0.5 \ldots 2.0) \times 2 \pi T$, with the variation providing an error band. The results are plotted in Fig. 2.

\section{CONCLUSIONS}

The goal of this technical contribution, motivated by the potential cosmological applications mentioned in Sec. I, has been to estimate a QCD Debye mass, defined as a matching coefficient of the dimensionally reduced effective theory, at temperatures between $1 \mathrm{GeV}$ and $10 \mathrm{TeV}$. For this purpose, we have carried out a two-loop computation, reducing the result to a small number of exponentially convergent one- and two-dimensional integrals, which are readily evaluated numerically.

The most nontrivial parts of our work established the IBP relations in Eqs. (3.13)-(3.15) and resolved the two-loop master sum integral $Z_{112}$ in Appendix A 2. With these ingredients, we obtain integral representations for the various functions parametrizing our result, cf. Eq. (3.18), which are shown in Eqs. (4.2)-(4.7) and evaluated numerically in Fig. 2 (left). Putting everything together and inserting the values of running Standard Model couplings, we find that quark mass thresholds are crossed smoothly enough not to be discernible by the bare eye, apart from that related to the top quark, cf. Fig. 2 (right).

The steps of the computation have been described on a detailed level, in order to permit the inclusion of further massive particles if present, such as of scalar fields. Hopefully, these results or techniques can find use, e.g., in dark matter computations involving strongly interacting coannihilation partners or in precision studies of the electroweak phase transition in extensions of the Standard Model. 


\section{ACKNOWLEDGMENTS}

This work was partly supported by the Swiss National Science Foundation (SNF) under Grant No. 200020B188712 and by the Chilean FONDECYT under Project No. 1191073.

\section{APPENDIX A: MASTER SUM INTEGRALS}

We list here the expressions for the master sum integrals appearing in Eqs. (3.19)-(3.25).

\section{One-loop structures}

We start by reiterating the expressions for a number of oneloop master sum integrals, defined according to Eq. (3.2). General techniques for evaluating massless sum integrals were developed in Refs. [39,40]. In the bosonic case,

$$
\begin{aligned}
\hat{Z}_{1 ; 0}= & \frac{T^{2}}{12}\left\{1+2 \epsilon\left[\ln \left(\frac{\bar{\mu} e^{\gamma_{\mathrm{E}}}}{4 \pi T}\right)+\ln (2 \pi)-\left(\ln \zeta_{2}\right)^{\prime}\right]\right. \\
& \left.+\mathcal{O}\left(\epsilon^{2}\right)\right\},
\end{aligned}
$$

where $\zeta_{n}=\zeta(n)$ is the Riemann zeta function, $\left(\ln \zeta_{n}\right)^{\prime} \equiv$ $\zeta^{\prime}(n) / \zeta(n)$, and $\bar{\mu}^{2} \equiv 4 \pi \mu^{2} e^{-\gamma_{\mathrm{E}}}$. In the literature, a different form is often shown, obtained by employing the identity $\ln (2 \pi)-\left(\ln \zeta_{2}\right)^{\prime}=1-\gamma_{\mathrm{E}}+\left(\ln \zeta_{-1}\right)^{\prime}$. A quadratic propagator similarly yields

$$
\begin{aligned}
\hat{Z}_{2 ; 0}= & \frac{1}{(4 \pi)^{2}}\left\{\frac{1}{\epsilon}+2 \ln \left(\frac{\bar{\mu} e^{\gamma_{\mathrm{E}}}}{4 \pi T}\right)\right. \\
& \left.+2 \epsilon\left[\ln ^{2}\left(\frac{\bar{\mu} e^{\gamma_{\mathrm{E}}}}{4 \pi T}\right)+\frac{\pi^{2}}{8}-\gamma_{\mathrm{E}}^{2}-2 \gamma_{1}\right]+\mathcal{O}\left(\epsilon^{2}\right)\right\},
\end{aligned}
$$

where $\gamma_{1}$ is a Stieltjes constant. More generally, $\hat{Z}_{j ; 0}=$ $\frac{\bar{\mu}^{3-d} \exp \left[(3-d) \gamma_{\mathrm{r}} / 2\right] \Gamma(j-d / 2) \zeta(2 j-d)}{8 \pi^{5 / 2}(2 \pi T)^{2 j-1-d} \Gamma(j)}$.

In the fermionic case, when the mass is nonzero, no analytic expressions are available. Even if convergent sum representations in terms of modified Bessel functions can be found, in practice, it is simpler to handle integral representations, such as

$$
\begin{aligned}
Z_{1 ; i}= & -\frac{m_{i}^{2}}{(4 \pi)^{2} \epsilon}-\frac{m_{i}^{2}}{(4 \pi)^{2}}\left[\ln \left(\frac{\bar{\mu}^{2}}{m_{i}^{2}}\right)+1\right]-\int_{p} \frac{n_{\mathrm{F}}\left(\omega_{p_{i}}\right)}{\omega_{p_{i}}} \\
& -\epsilon\left\{\frac{m_{i}^{2}}{(4 \pi)^{2}}\left[\frac{1}{2} \ln ^{2}\left(\frac{\bar{\mu}^{2}}{m_{i}^{2}}\right)+\ln \left(\frac{\bar{\mu}^{2}}{m_{i}^{2}}\right)+1+\frac{\pi^{2}}{12}\right]+\int_{p} \frac{n_{\mathrm{F}}\left(\omega_{p_{i}}\right)}{\omega_{p_{i}}}\left[\ln \left(\frac{\bar{\mu}^{2}}{4 p^{2}}\right)+2\right]\right\}+\mathcal{O}\left(\epsilon^{2}\right),
\end{aligned}
$$

where $\int_{p} \equiv \int \frac{\mathrm{d}^{3} \mathbf{p}}{(2 \pi)^{3}}$ and $\omega_{p_{i}} \equiv \sqrt{p^{2}+m_{i}^{2}}$. Taking a mass derivative and carrying out a partial integration gives

$$
\begin{aligned}
Z_{2 ; i}= & \frac{1}{(4 \pi)^{2} \epsilon}+\frac{1}{(4 \pi)^{2}} \ln \left(\frac{\bar{\mu}^{2}}{m_{i}^{2}}\right)-\int_{p} \frac{n_{\mathrm{F}}\left(\omega_{p_{i}}\right)}{2 p^{2} \omega_{p_{i}}} \\
& +\epsilon\left\{\frac{1}{(4 \pi)^{2}}\left[\frac{1}{2} \ln ^{2}\left(\frac{\bar{\mu}^{2}}{m_{i}^{2}}\right)+\frac{\pi^{2}}{12}\right]-\int_{p} \frac{n_{\mathrm{F}}\left(\omega_{p_{i}}\right)}{2 p^{2} \omega_{p_{i}}} \ln \left(\frac{\bar{\mu}^{2}}{4 p^{2}}\right)\right\}+\mathcal{O}\left(\epsilon^{2}\right) .
\end{aligned}
$$

One more mass derivative yields (this time no partial integration is possible; $\beta \equiv 1 / T$ )

$$
\begin{aligned}
Z_{3 ; i}= & \frac{1}{(4 \pi)^{2} 2 m_{i}^{2}}-\int_{p} \frac{n_{\mathrm{F}}\left(\omega_{p_{i}}\right)+\beta \omega_{p_{i}} n_{\mathrm{F}}\left(\omega_{p_{i}}\right)\left[1-n_{\mathrm{F}}\left(\omega_{p_{i}}\right)\right]}{8 p^{2} \omega_{p_{i}}^{3}} \\
& +\epsilon\left\{\frac{\ln \left(\bar{\mu}^{2} / m_{i}^{2}\right)}{(4 \pi)^{2} 2 m_{i}^{2}}-\int_{p} \frac{n_{\mathrm{F}}\left(\omega_{p_{i}}\right)+\beta \omega_{p_{i}} n_{\mathrm{F}}\left(\omega_{p_{i}}\right)\left[1-n_{\mathrm{F}}\left(\omega_{p_{i}}\right)\right]}{p^{2} \omega_{p_{i}}^{3}} \ln \left(\frac{\bar{\mu}^{2}}{4 p^{2}}\right)\right\}+\mathcal{O}\left(\epsilon^{2}\right) .
\end{aligned}
$$

\section{Two-loop master $Z_{112}$}

Even if in the massless limit IBP identities allow one to reduce $Z_{112}$ as

$$
\lim _{m \rightarrow 0} Z_{112}=\lim _{m_{i} \rightarrow 0} \frac{Z_{2 ; i}\left(Z_{2 ; i}-2 \hat{Z}_{2 ; 0}\right)}{(d-2)(d-5)}
$$

no such factorization has been found for $m \neq 0$. The result for a fully massive $Z_{111}$ is given in Ref. [41], and one might think that $Z_{112}$ could be obtained as a mass derivative thereof; however, this does not work trivially as setting the third mass to zero after the derivative leads to IR divergences (linear and logarithmic). A careful consideration is thus needed for working out the reduction 
of $Z_{112}$ into a convergent two-dimensional integral representation.

For a first step, let us carry out the Matsubara sums. The quadratic propagator carries a fictitious mass parameter, denoted by $M^{2}$, as an intermediate regulator. The sum integral splits into a vacuum part, one-cut parts, and two-cut parts, with "cut" meaning that some line is put on shell and weighted by a thermal distribution:

$$
\begin{aligned}
Z_{112} & =Z_{112}^{(\mathrm{vac})}+Z_{112}^{(\mathrm{B})}+Z_{112}^{(\mathrm{F})}+Z_{112}^{(\mathrm{FB})}+Z_{112}^{(\mathrm{FF})}, \\
Z_{112}^{(\mathrm{vac})} & =\int_{P, Q} \frac{1}{\left(P^{2}+m^{2}\right)\left(Q^{2}+m^{2}\right)(P-Q)^{4}}, \\
Z_{112}^{(\mathrm{B})} & =-\lim _{M \rightarrow 0} \frac{\mathrm{d}}{\mathrm{d} M^{2}} \int_{p} \frac{n_{\mathrm{B}}\left(\Omega_{p}\right)}{\Omega_{p}}\left[\int_{Q} \frac{1}{\left(Q^{2}+m^{2}\right)\left[(P-Q)^{2}+m^{2}\right]}\right]_{P^{2}=-M^{2}}, \\
Z_{112}^{(\mathrm{F})} & =-2 \lim _{M \rightarrow 0} \int_{p} \frac{n_{\mathrm{F}}\left(\omega_{p}\right)}{\omega_{p}}\left[\int_{Q} \frac{1}{\left(Q^{2}+M^{2}\right)^{2}\left[(P-Q)^{2}+m^{2}\right]}\right]_{P^{2}=-m^{2}}, \\
Z_{112}^{(\mathrm{FB})} & =2 \lim _{M \rightarrow 0} \frac{\mathrm{d}}{\mathrm{d} M^{2}} \int_{p, q} \frac{n_{\mathrm{F}}\left(\omega_{p}\right) n_{\mathrm{B}}\left(\Omega_{q}\right)}{\omega_{p} \Omega_{q}}\left[\frac{1}{(P-Q)^{2}+m^{2}}\right]_{P^{2}=-m^{2}, Q^{2}=-M^{2}} \\
Z_{112}^{(\mathrm{FF})} & =\lim _{M \rightarrow 0} \int_{p, q} \frac{n_{\mathrm{F}}\left(\omega_{p}\right) n_{\mathrm{F}}\left(\omega_{q}\right)}{\omega_{p} \omega_{q}}\left[\frac{1}{\left[(P-Q)^{2}+M^{2}\right]^{2}}\right]_{P^{2}=-m^{2}, Q^{2}=-m^{2}} .
\end{aligned}
$$

Here, $\Omega_{p} \equiv \sqrt{p^{2}+M^{2}}, \omega_{p} \equiv \sqrt{p^{2}+m^{2}}, \int_{P} \equiv \mu^{3-d} \int \frac{\mathrm{d}^{d+1} P}{(2 \pi)^{d+1}}$, and $\int_{p} \equiv \mu^{3-d} \int \frac{\mathrm{d}^{d} \mathbf{p}}{(2 \pi)^{d}}$. The cuts are

$$
[\ldots]_{P^{2}=-m^{2}} \equiv \frac{1}{2} \sum_{p_{n}= \pm i \omega_{p}}[\ldots], \quad[\ldots]_{P^{2}=-m^{2}, Q^{2}=-M^{2}} \equiv \frac{1}{4} \sum_{p_{n}= \pm i \omega_{p}} \sum_{q_{n}= \pm i \Omega_{q}}[\ldots] .
$$

The parameter $M$ is set to zero for the final spatial integrals, which are treated with strict dimensional regularization (this is necessary, given that the IBP identities used for reducing the result to this basis made use of the same recipe).

Two of the structures in Eq. (A7) are simple to handle, namely, the vacuum part and the one-cut part with a single Bose distribution:

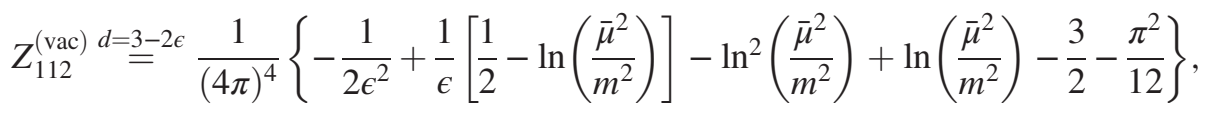

$$
\begin{aligned}
& Z_{112}^{(\mathrm{B}) d=3-2 \epsilon}=\frac{\hat{Z}_{1 ; 0}}{6 m^{2}(4 \pi)^{2}}+\frac{\hat{Z}_{2 ; 0}}{(4 \pi)^{2}}\left\{\frac{1}{\epsilon}+\ln \left(\frac{\bar{\mu}^{2}}{m^{2}}\right)+\epsilon\left[\frac{1}{2} \ln ^{2}\left(\frac{\bar{\mu}^{2}}{m^{2}}\right)+\frac{\pi^{2}}{12}\right]\right\} \text {. }
\end{aligned}
$$

The remaining parts are more subtle, as they are IR divergent, in a way which is not trivially handled by dimensional regularization.

Let us start by considering $Z_{112}^{(\mathrm{FF})}$, which contains a linear IR divergence but no logarithmic one. As this integral is UV finite, we may set $d=3$ and carry out the angular integral, which yields

$$
Z_{112}^{(\mathrm{FF})} \stackrel{d=3}{\simeq} \frac{1}{4 m^{2}} \int_{p, q} \frac{n_{\mathrm{F}}\left(\omega_{p}\right) n_{\mathrm{F}}\left(\omega_{q}\right)}{\omega_{p} \omega_{q}} \frac{\omega_{p}^{2}+\omega_{q}^{2}}{\left(\omega_{p}^{2}-\omega_{q}^{2}\right)^{2}} .
$$

Clearly, this is ill defined around $p=q$. To find a useful representation, we make use of symmetries of the integrand, reorganizing the Fermi distributions as

$$
\begin{aligned}
& Z_{112}^{(\mathrm{FF})} \stackrel{d=3}{=} \frac{1}{16 m^{2}} \int_{p, q} \frac{1}{\omega_{p} \omega_{q}}\left\{\frac{\left[n_{\mathrm{F}}\left(\omega_{p}\right)+n_{\mathrm{F}}\left(\omega_{q}\right)\right]^{2}}{\left(\omega_{p}+\omega_{q}\right)^{2}}-\frac{\left[n_{\mathrm{F}}\left(\omega_{p}\right)-n_{\mathrm{F}}\left(\omega_{q}\right)\right]^{2}}{\left(\omega_{p}-\omega_{q}\right)^{2}}\right\}+\delta Z_{112}^{(\mathrm{FF})}, \\
& \delta Z_{112}^{(\mathrm{FF})} \stackrel{d=3}{=} \int_{p} \frac{n_{\mathrm{F}}^{2}\left(\omega_{p}\right)}{2 m^{2}} \int_{q} \frac{1}{\left(q^{2}-p^{2}\right)^{2}}
\end{aligned}
$$


Considering the vacuumlike integral in Eq. (A18) as an analytic function of $-p^{2}$ and taking the real part yields $\operatorname{Re} \int_{q} \frac{1}{\left(q^{2}-p^{2}\right)^{2}}=\operatorname{Re} \frac{1}{8 \pi\left(-p^{2}\right)^{1 / 2}}=0$. Alternatively, if we keep the regulator $M$ finite, $\delta Z_{112}^{(\mathrm{FF})}$ contains a linear divergence ${ }^{6}$ proportional to $1 / M$ but no logarithmic or finite part of $\mathcal{O}\left(M^{0}\right)$. To summarize, in strict dimensional regularization, we can set $\delta Z_{112}^{(\mathrm{FF})} \rightarrow 0$.
The remaining parts, $Z_{112}^{(\mathrm{F})}$ and $Z_{112}^{(\mathrm{FB})}$, contain both linear and logarithmic divergences. The logarithmic divergences cancel in the sum. We find it practical to determine the sum by keeping $M$ finite and taking $M \rightarrow 0$ at the end, omitting again linear divergences proportional to $1 / M$, which are absent in strict dimensional regularization. A rather tedious analysis then yields

$$
Z_{112}^{(\mathrm{F})}+Z_{112}^{(\mathrm{FB}) d=3} \frac{2}{m^{2}(4 \pi)^{2}} \int_{p} \frac{n_{\mathrm{F}}\left(\omega_{p}\right)}{\omega_{p}}\left[\ln \left(\frac{m e^{\gamma_{\mathrm{E}}}}{4 \pi T}\right)+1+\frac{\omega_{p}}{2 p} \ln \left(\frac{\omega_{p}+p}{\omega_{p}-p}\right)\right] .
$$

Given the nontriviality of the steps, it is good to check that Eq. (A6) is correctly reproduced for $m / T \rightarrow 0$. The individual parts contain coefficients $\propto 1 / \mathrm{m}^{2}$, so we need to expand to $\mathcal{O}\left(\mathrm{m}^{2}\right)$. The integral appearing in Eq. (A19) can be expanded as

$$
\begin{gathered}
\int_{p} \frac{n_{\mathrm{F}}\left(\omega_{p}\right)}{\omega_{p}}\left[\ln \left(\frac{m e^{\gamma_{\mathrm{E}}}}{4 \pi T}\right)+1+\frac{\omega_{p}}{2 p} \ln \left(\frac{\omega_{p}+p}{\omega_{p}-p}\right)\right]=\frac{T^{2}}{24}\left[2+\left(\ln \zeta_{2}\right)^{\prime}-\ln \pi\right] \\
+\frac{2 m^{2}}{(4 \pi)^{2}}\left[\ln ^{2}\left(\frac{m e^{\gamma_{\mathrm{E}}}}{4 \pi T}\right)+(1+2 \ln 2) \ln \left(\frac{m e^{\gamma_{\mathrm{E}}}}{4 \pi T}\right)+3 \ln 2-\frac{1}{2}\right]+\mathcal{O}\left(m^{4}\right),
\end{gathered}
$$

whereas the contribution from Eq. (A17) can be numerically verified to behave as

$$
\begin{aligned}
\int_{p, q} & \frac{1}{\omega_{p} \omega_{q}}\left\{\frac{\left[n_{\mathrm{F}}\left(\omega_{p}\right)+n_{\mathrm{F}}\left(\omega_{q}\right)\right]^{2}}{\left(\omega_{p}+\omega_{q}\right)^{2}}-\frac{\left[n_{\mathrm{F}}\left(\omega_{p}\right)-n_{\mathrm{F}}\left(\omega_{q}\right)\right]^{2}}{\left(\omega_{p}-\omega_{q}\right)^{2}}\right\}=-\frac{4 T^{2}}{3(4 \pi)^{2}}\left[\frac{11}{6}+\left(\ln \zeta_{2}\right)^{\prime}-\ln \pi\right] \\
- & \frac{32 m^{2}}{(4 \pi)^{4}}\left[\ln ^{2}\left(\frac{m e^{\gamma_{\mathrm{E}}}}{\pi T}\right)+\ln \left(\frac{m e^{\gamma_{\mathrm{E}}}}{\pi T}\right)+4 \ln 2-\frac{5}{2}\right]+\mathcal{O}\left(m^{4}\right) .
\end{aligned}
$$

Summing together and adding the other parts, we reproduce the result from Eq. (A6),

$$
\lim _{m \rightarrow 0} Z_{112} \stackrel{d=3-2 \epsilon}{=} \frac{1}{(4 \pi)^{4}}\left\{\frac{1}{2 \epsilon^{2}}+\frac{1}{\epsilon}\left[\frac{1}{2}+2 \ln \left(\frac{\bar{\mu} e^{\gamma_{\mathrm{E}}}}{4 \pi T}\right)\right]+4 \ln ^{2}\left(\frac{\bar{\mu} e^{\gamma_{\mathrm{E}}}}{4 \pi T}\right)+2 \ln \left(\frac{\bar{\mu} e^{\gamma_{\mathrm{E}}}}{4 \pi T}\right)-8 \ln ^{2} 2+\frac{\pi^{2}}{4}+\frac{3}{2}-2 \gamma_{\mathrm{E}}^{2}-4 \gamma_{1}\right\} .
$$

\section{Two-loop master $Z_{212}$}

In the massless limit, IBP identities allow one to reduce $Z_{212}$ as

$$
\lim _{m \rightarrow 0} Z_{212}=\lim _{m_{i} \rightarrow 0} \frac{2 Z_{3 ; i}\left(Z_{2 ; i}-\hat{Z}_{2 ; 0}\right)}{(d-2)(d-7)} .
$$

For a finite mass, we can instead write

$$
Z_{212}=-\frac{1}{2} \frac{\mathrm{d} Z_{112}}{\mathrm{~d} m^{2}}
$$

Converting a number of mass derivatives into derivatives with respect to momentum, and carrying out partial integrations, Eqs. (A14), (A15), (A17), and (A19) then imply that

$$
\begin{aligned}
Z_{212} \stackrel{d=3-2 \epsilon}{=} & -\frac{1}{2 m^{2}(4 \pi)^{4}}\left[\frac{1}{\epsilon}+2 \ln \left(\frac{\bar{\mu}^{2}}{m^{2}}\right)-1\right]-\frac{\hat{Z}_{1 ; 0}}{12 m^{4}(4 \pi)^{2}}+\frac{\hat{Z}_{2 ; 0}}{2 m^{2}(4 \pi)^{2}}\left[1+\epsilon \ln \left(\frac{\bar{\mu}^{2}}{m^{2}}\right)\right] \\
& +\frac{1}{m^{4}(4 \pi)^{2}} \int_{p} \frac{n_{\mathrm{F}}\left(\omega_{p}\right)}{\omega_{p}}\left\{\left(1+\frac{m^{2}}{2 p^{2}}\right) \ln \left(\frac{m e^{\gamma \mathrm{E}}}{4 \pi T}\right)+\frac{\omega_{p}^{2}}{p^{2}}+\frac{\omega_{p}}{2 p} \ln \left(\frac{\omega_{p}+p}{\omega_{p}-p}\right)\right\} \\
& +\frac{1}{64 m^{4}} \int_{p, q} \frac{1}{\omega_{p} \omega_{q}}\left(\frac{\omega_{p}^{2}}{p^{2}}+\frac{\omega_{q}^{2}}{q^{2}}\right)\left\{\frac{\left[n_{\mathrm{F}}\left(\omega_{p}\right)+n_{\mathrm{F}}\left(\omega_{q}\right)\right]^{2}}{\left(\omega_{p}+\omega_{q}\right)^{2}}-\frac{\left[n_{\mathrm{F}}\left(\omega_{p}\right)-n_{\mathrm{F}}\left(\omega_{q}\right)\right]^{2}}{\left(\omega_{p}-\omega_{q}\right)^{2}}\right\} .
\end{aligned}
$$

\footnotetext{
${ }^{6}$ The sum of all $1 / M$ divergences in $Z_{112}$ equals the Matsubara zero-mode contribution $T \int_{p} \frac{1}{\left(p^{2}+M^{2}\right)^{2}} Z_{2 ; i}$.
} 
An interesting cross-check of Eq. (A25) can be obtained by considering the massless limit. As there are coefficients proportional to $1 / \mathrm{m}^{4}$, we need to expand the integrals up to $\mathcal{O}\left(\mathrm{m}^{4}\right)$,

$$
\begin{aligned}
& \int_{p} \frac{n_{\mathrm{F}}\left(\omega_{p}\right)}{\omega_{p}}\left\{\left(1+\frac{m^{2}}{2 p^{2}}\right) \ln \left(\frac{m e^{\gamma_{\mathrm{E}}}}{4 \pi T}\right)+\frac{\omega_{p}^{2}}{p^{2}}+\frac{\omega_{p}}{2 p} \ln \left(\frac{\omega_{p}+p}{\omega_{p}-p}\right)\right\} \\
& =\frac{T^{2}}{24}\left[2+\left(\ln \zeta_{2}\right)^{\prime}-\ln \pi\right]-\frac{2 m^{2}}{(4 \pi)^{2}}\left[\ln \left(\frac{m e^{\gamma_{\mathrm{E}}}}{4 \pi T}\right)+\ln 2+\frac{1}{2}\right]+\frac{14 \zeta_{3} m^{4}}{(4 \pi)^{4} T^{2}}\left[\ln \left(\frac{m e^{\gamma_{\mathrm{E}}}}{4 \pi T}\right)+\frac{9}{4}\right]+\mathcal{O}\left(m^{6}\right), \\
& \frac{1}{64} \int_{p, q} \frac{1}{\omega_{p} \omega_{q}}\left(\frac{\omega_{p}^{2}}{p^{2}}+\frac{\omega_{q}^{2}}{q^{2}}\right)\left\{\frac{\left[n_{\mathrm{F}}\left(\omega_{p}\right)+n_{\mathrm{F}}\left(\omega_{q}\right)\right]^{2}}{\left(\omega_{p}+\omega_{q}\right)^{2}}-\frac{\left[n_{\mathrm{F}}\left(\omega_{p}\right)-n_{\mathrm{F}}\left(\omega_{q}\right)\right]^{2}}{\left(\omega_{p}-\omega_{q}\right)^{2}}\right\} \\
& =-\frac{T^{2}}{24(4 \pi)^{2}}\left[\frac{11}{6}+\left(\ln \zeta_{2}\right)^{\prime}-\ln \pi\right]+\frac{m^{2}}{(4 \pi)^{4}}\left[\ln \left(\frac{m e^{\gamma_{\mathrm{E}}}}{\pi T}\right)+\frac{1}{2}\right]-\frac{14 \zeta_{3} m^{4}}{(4 \pi)^{6} T^{2}}\left[\ln \left(\frac{m e^{\gamma_{\mathrm{E}}}}{\pi T}\right)+\frac{9}{4}\right]+\mathcal{O}\left(m^{6}\right),
\end{aligned}
$$

the last of which was verified numerically. Summing together and adding the other terms, we recover the result from Eq. (A23),

$$
\lim _{m \rightarrow 0} Z_{212} \stackrel{d=3-2 \epsilon}{=}-\frac{28 \zeta_{3} \ln 2}{(4 \pi)^{6} T^{2}}
$$

[1] M. Garny, J. Heisig, M. Hufnagel, and B. Lülf, Top-philic dark matter within and beyond the WIMP paradigm, Phys. Rev. D 97, 075002 (2018).

[2] S. Kim and M. Laine, On thermal corrections to nearthreshold annihilation, J. Cosmol. Astropart. Phys. 01 (2017) 013.

[3] D. Bödeker and M. Wörmann, Non-relativistic leptogenesis, J. Cosmol. Astropart. Phys. 02 (2014) 016.

[4] P. B. Arnold, S. Cantrell, and W. Xiao, Stopping distance for high energy jets in weakly-coupled quark-gluon plasmas, Phys. Rev. D 81, 045017 (2010).

[5] S. Caron-Huot, $O(g)$ plasma effects in jet quenching, Phys. Rev. D 79, 065039 (2009).

[6] K. Kajantie, M. Laine, K. Rummukainen, and M. E. Shaposhnikov, Generic rules for high temperature dimensional reduction and their application to the Standard Model, Nucl. Phys. B458, 90 (1996).

[7] D. Bödeker, P. John, M. Laine, and M. G. Schmidt, The 2-loop MSSM finite temperature effective potential with stop condensation, Nucl. Phys. B497, 387 (1997).

[8] H. H. Patel, M. J. Ramsey-Musolf, and M. B. Wise, Color breaking in the early universe, Phys. Rev. D 88, 015003 (2013).

[9] J. I. Kapusta, Quantum chromodynamics at high temperature, Nucl. Phys. B148, 461 (1979).

[10] P. B. Arnold and L. G. Yaffe, The non-Abelian Debye screening length beyond leading order, Phys. Rev. D 52, 7208 (1995).

[11] A. K. Rebhan, Non-Abelian Debye mass at next-to-leading order, Phys. Rev. D 48, R3967 (1993).

[12] Y. Burnier and A. Rothkopf, A gauge invariant Debye mass and the complex heavy-quark potential, Phys. Lett. B 753, 232 (2016).
[13] P. H. Ginsparg, First and second order phase transitions in gauge theories at finite temperature, Nucl. Phys. B170, 388 (1980).

[14] T. Appelquist and R. D. Pisarski, High-temperature YangMills theories and three-dimensional Quantum Chromodynamics, Phys. Rev. D 23, 2305 (1981).

[15] M. Laine and M. Vepsäläinen, On the smallest screening masses in hot QCD, J. High Energy Phys. 09 (2009) 023.

[16] J. Möller and Y. Schröder, Three-loop matching coefficients for hot QCD: Reduction and gauge independence, J. High Energy Phys. 08 (2012) 025.

[17] I. Ghişoiu, J. Möller, and Y. Schröder, Debye screening mass of hot Yang-Mills theory to three-loop order, J. High Energy Phys. 11 (2015) 121.

[18] E. Braaten and A. Nieto, Free energy of QCD at high temperature, Phys. Rev. D 53, 3421 (1996).

[19] I. Ghişoiu, Three-loop Debye mass and effective coupling in thermal QCD, Ph.D. thesis, University of Bielefeld, 2013, https://pub.uni-bielefeld.de/publication/2632705.

[20] I. Ghişoiu and Y. Schröder, Poster presentation at the Latsis EPFL Symposium SEWM14, Lausanne, Switzerland, 2014, http://www.sewm14.unibe.ch/ghisoiu.pdf.

[21] M. Laine, P. Schicho, and Y. Schröder, Soft thermal contributions to 3-loop gauge coupling, J. High Energy Phys. 05 (2018) 037.

[22] M. Laine and Y. Schröder, Two-loop QCD gauge coupling at high temperatures, J. High Energy Phys. 03 (2005) 067.

[23] M. Laine and Y. Schröder, Quark mass thresholds in QCD thermodynamics, Phys. Rev. D 73, 085009 (2006).

[24] L.F. Abbott, The background field method beyond one loop, Nucl. Phys. B185, 189 (1981). 
[25] K. G. Chetyrkin and F. V. Tkachov, Integration by parts: The algorithm to calculate $\beta$-functions in 4 loops, Nucl. Phys. B192, 159 (1981).

[26] M. Nishimura and Y. Schröder, IBP methods at finite temperature, J. High Energy Phys. 09 (2012) 051.

[27] J. Kuipers, T. Ueda, J. A. M. Vermaseren, and J. Vollinga, FORM version 4.0, Comput. Phys. Commun. 184, 1453 (2013).

[28] S. Laporta, High precision calculation of multiloop Feynman integrals by difference equations, Int. J. Mod. Phys. A 15, 5087 (2000).

[29] M. Tanabashi et al. (Particle Data Group), Review of particle physics, Phys. Rev. D 98, 030001 (2018).

[30] P. A. Baikov, K. G. Chetyrkin, and J. H. Kühn, Five-Loop Running of the QCD Coupling Constant, Phys. Rev. Lett. 118, 082002 (2017).

[31] F. Herzog, B. Ruijl, T. Ueda, J. A. M. Vermaseren, and A. Vogt, The five-loop beta function of Yang-Mills theory with fermions, J. High Energy Phys. 02 (2017) 090.

[32] T. Luthe, A. Maier, P. Marquard, and Y. Schröder, The fiveloop Beta function for a general gauge group and anomalous dimensions beyond Feynman gauge, J. High Energy Phys. 10 (2017) 166.

[33] K. G. Chetyrkin, G. Falcioni, F. Herzog, and J. A. M. Vermaseren, Five-loop renormalisation of QCD in covariant gauges, J. High Energy Phys. 10 (2017) 179; Addendum, J. High Energy Phys. 12 (2017) 006.

[34] K. G. Chetyrkin and M. F. Zoller, Three-loop $\beta$-functions for top-Yukawa and the Higgs self-interaction in the Standard Model, J. High Energy Phys. 06 (2012) 033.

[35] L. N. Mihaila, J. Salomon, and M. Steinhauser, Renormalization constants and beta functions for the gauge couplings of the Standard Model to three-loop order, Phys. Rev. D 86, 096008 (2012).

[36] P. A. Baikov, K. G. Chetyrkin, and J. H. Kühn, Quark mass and field anomalous dimensions to $\mathcal{O}\left(\alpha_{s}^{5}\right)$, J. High Energy Phys. 10 (2014) 076.

[37] T. Luthe, A. Maier, P. Marquard, and Y. Schröder, Five-loop quark mass and field anomalous dimensions for a general gauge group, J. High Energy Phys. 01 (2017) 081.

[38] M. D'Onofrio and K. Rummukainen, Standard Model cross-over on the lattice, Phys. Rev. D 93, 025003 (2016).

[39] P. B. Arnold and C. Zhai, The three loop free energy for pure gauge QCD, Phys. Rev. D 50, 7603 (1994).

[40] P. B. Arnold and C. Zhai, The three loop free energy for high temperature QED and QCD with fermions, Phys. Rev. D 51, 1906 (1995).

[41] M. Laine, M. Meyer, and G. Nardini, Thermal phase transition with full 2-loop effective potential, Nucl. Phys. B920, 565 (2017). 